\title{
Adrenal Schwannoma in an Elderly Man: A Case Report and Literature Review
}

\author{
Kenji Yorita ${ }^{1}$, Takushi Naroda ${ }^{2}$, Masato Tamura ${ }^{2}$, Satoshi Ito $^{3}$ and Kimiko Nakatani ${ }^{3}$
}

\begin{abstract}
:
Schwannoma is a common mesenchymal neoplasm; however, adrenal schwannoma is rare, and it is frequently misdiagnosed as adrenal cortical adenoma. We herein report a 91-year-old Japanese man with right adrenal schwannoma that was pathologically diagnosed after adrenalectomy. To our knowledge, this is the first case of adrenal schwannoma in the oldest patient and with the longest follow-up period reported, including radiological images from 10 years earlier.
\end{abstract}

Key words: Schwannoma, adrenal gland, elderly man

(Intern Med Advance Publication)

(DOI: 10.2169/internalmedicine.7026-21)

\section{Background}

Schwannoma is a common benign peripheral nerve sheath tumor derived from Schwann cells, including those of the myelinic sheath of peripheral autonomic or cranial nerves. The tumor can arise in any area of neural tissue, including Schwann cells; however, the common tumor sites are the skin and subcutaneous tissue of the head and neck or flexor surfaces of the extremities. Adrenal schwannoma is rare and frequently misdiagnosed as adrenal cortical adenoma (1).

We encountered a 91-year-old Japanese man with right adrenal schwannoma. We believe that this is the oldest case of adrenal schwannoma with the longest follow-up period $(1,2)$. We performed radiological evaluations, including ${ }^{18} \mathrm{~F}$-2-fluoro-2-deoxy-D-glucose (FDG) positron emission tomography (PET), which is rare in cases of adrenal schwannomas. We herein report the clinicopathological aspects of the tumor.

\section{Case Presentation}

\section{Clinical summary}

A 90-year-old asymptomatic man was admitted for clinical management of a right adrenal gland tumor after the tu- mor had been incidentally found on computed tomography (CT) 1 year earlier. He had a clinical history of cerebral infarction and carotid endarterectomy. Neurofibromatosis type 2 was deemed unlikely due to the absence of acoustic schwannoma and meningioma.

The results of laboratory tests, including those for measuring plasma levels of adrenocorticotrophic hormone, cortisol, aldosterone, adrenaline, noradrenaline, dehydroepiandrosterone sulfate, and dopamine as well as urine levels of adrenaline, noradrenaline, dopamine, vanillylmandelic acid, and homovanillic acid, were within normal limits. Plasma renin activity was also normal. The levels of serum markers for carcinoma were within normal limits as well, except for squamous cell carcinoma antigen $(1.7 \mathrm{ng} / \mathrm{mL}$, normal range $<1.5 \mathrm{ng} / \mathrm{mL}$ ). High serum levels of soluble interleukin 2 receptors were found $(851 \mathrm{U} / \mathrm{mL}$, normal range 122-496 U/ $\mathrm{mL})$.

Non-enhanced CT performed 1 year earlier showed a mass with a maximum diameter of $3.4 \mathrm{~cm}$, good demarcation, and a low density of 28.6 Hounsfield units (HU) compared to that in the liver (Fig. 1A). Magnetic resonance imaging (MRI) showed a heterogeneous, low-intensity signal on T1-weighted images (Fig. 1C) and high-intensity signal on T2-weighted images (Fig. 1D), and fatty tissue did not appear to be involved on chemical-shift MRI (CSMRI) (Fig. 1E) or fat-suppressed T2-weighted images (Fig. 1F).

${ }^{1}$ Department of Diagnostic Pathology, Japanese Red Cross Kochi Hospital, Kochi-city, Japan, ${ }^{2}$ Department of Urology, Japanese Red Cross Kochi Hospital, Kochi-city, Japan and ${ }^{3}$ Department of Radiology, Japanese Red Cross Kochi Hospital, Kochi-city, Japan

Received: January 2, 2021; Accepted: May 17, 2021; Advance Publication by J-STAGE: June 26, 2021

Correspondence to Dr. Kenji Yorita, kenjiyorita@gmail.com 

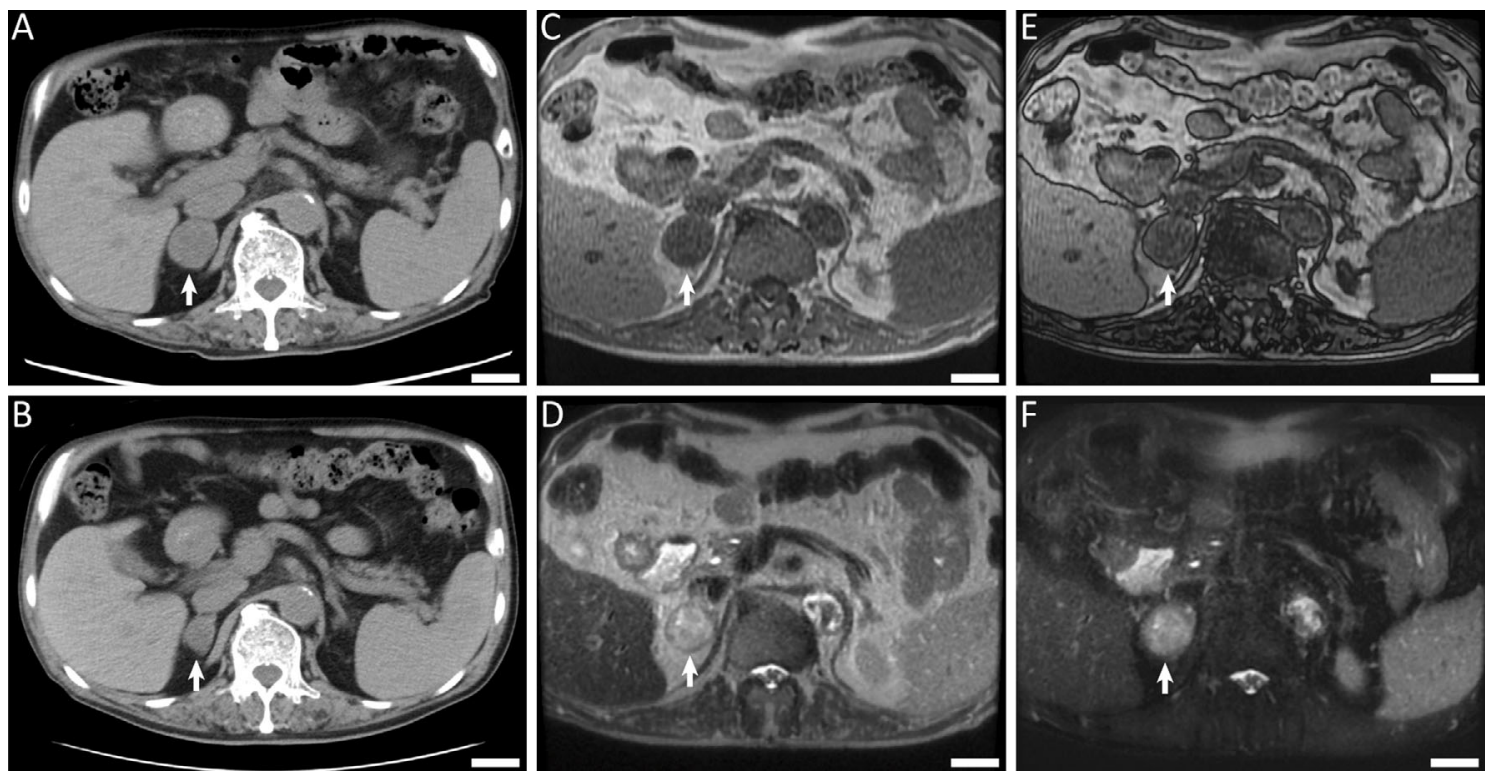

Figure 1. Computed tomography and magnetic resonance images of the adrenal schwannoma. One year earlier, a right adrenal tumor (white arrow) with a maximum size of $3.4 \mathrm{~cm}$ could be seen on computed tomography (CT) images (A). The tumor (white arrow) measured $2.2 \mathrm{~cm} 4$ years earlier on CT images (B). Magnetic resonance images taken one year earlier showed that the right adrenal tumor had a low intensity on T1-weighted (in-phase) images (C), had a high intensity on T2-weighted images (D), and was fat poor on opposed-phase T1-weighted images (E) and fat-suppressed T2weighted images (F). Scale bars represent $3 \mathrm{~cm}$.

FDG-PET showed the accumulation of FDG in the adrenal tumor (maximum standardized uptake value [SUVmax]: 4.4), but no abnormal FDG uptake was observed in other organs. A retrospective analysis of previous CT images revealed a suspected right adrenal gland tumor 10 years earlier, which had appeared as a lesion measuring $1.3 \mathrm{~cm} 7$ years earlier before gradually increasing in size to $2.2 \mathrm{~cm}$ (Fig. 1B) and $3.4 \mathrm{~cm}$ at 4 and 1 year earlier, respectively. The mass consistently showed good demarcation and a homogenous appearance. Clinically, a non-functioning adrenal tumor was found, and lipid-poor adrenal cortical adenoma (ACA), adrenal cortical carcinoma (ACC), and lymphoma were suspected.

After discussing the clinical management of the right adrenal tumor with the patient and his family, surgical treatment was chosen. However, surgery was postponed for approximately one year for several reasons. Although preoperative CT showed that the size of the tumor was unchanged compared to that 1 year earlier, right adrenalectomy was performed after further discussion with the 91-year-old patient and his family. Nine days after surgery, the patient was discharged without any problems.

\section{Macroscopic and microscopic findings of the adre- nal gland tumor}

Grossly, a well-demarcated and solid mass measuring 3.2 $\times 2.8 \times 2.8 \mathrm{~cm}$ was seen in the right adrenal gland (Fig. 2A). The cut surface of the tumor was white and yellow, and the thin brown line suggested that the adrenal cortex was located near the periphery of the tumor (Fig. 2B). Histologi- cally, the demarcated mass mostly consisted of an interlacing arrangement of spindle cells with plump elongated nuclei (Fig. 2C). Mitosis was rarely observed. Nuclear palisading of the tumor cells was noted (Fig. 2C), and Verocay bodies were also observed. Necrosis was absent. Hypocellular edematous areas were focally intermingled (Fig. 2D). The periphery of the tumor showed fibrosis and lymphoid tissue (Fig. 2E). Ganglion cells were not present in the tumor. Schwannoma was considered, and immunohistochemical studies supported the histological diagnosis; the tumor cells were diffusely positive for S100 protein (Fig. 2F) and SOX10 and negative for alpha-smooth muscle actin, desmin, EMA, neurofilament protein, KIT, and CD34. The Ki-67 labeling index was $1-2 \%$. The tumor was largely surrounded by the adrenal cortex (Fig. 2B, indicated by arrows), which was histologically present around approximately $80 \%$ of the tumor periphery. The adrenal medulla was focally present between the tumor and the adrenal cortex. The tumor cells proliferating outside the adrenal gland were inconspicuous. Thus, we concluded that the tumor was an adrenal schwannoma.

The adrenal cortex adjacent to the tumor was thin because the cortical thickness was $<1 \mathrm{~mm}$. The atrophic changes in the adrenal cortex might have been due to hormonal stimulation by the tumor cells. However, the serum and urine levels of hormones related to the adrenal gland were within normal ranges, and the histological proportion of the three layers of the adrenal cortex remained normal. Thus, the thin adrenal cortex appeared to be stretched by the tumor rather than by hormonal stimulation by tumor cells. 


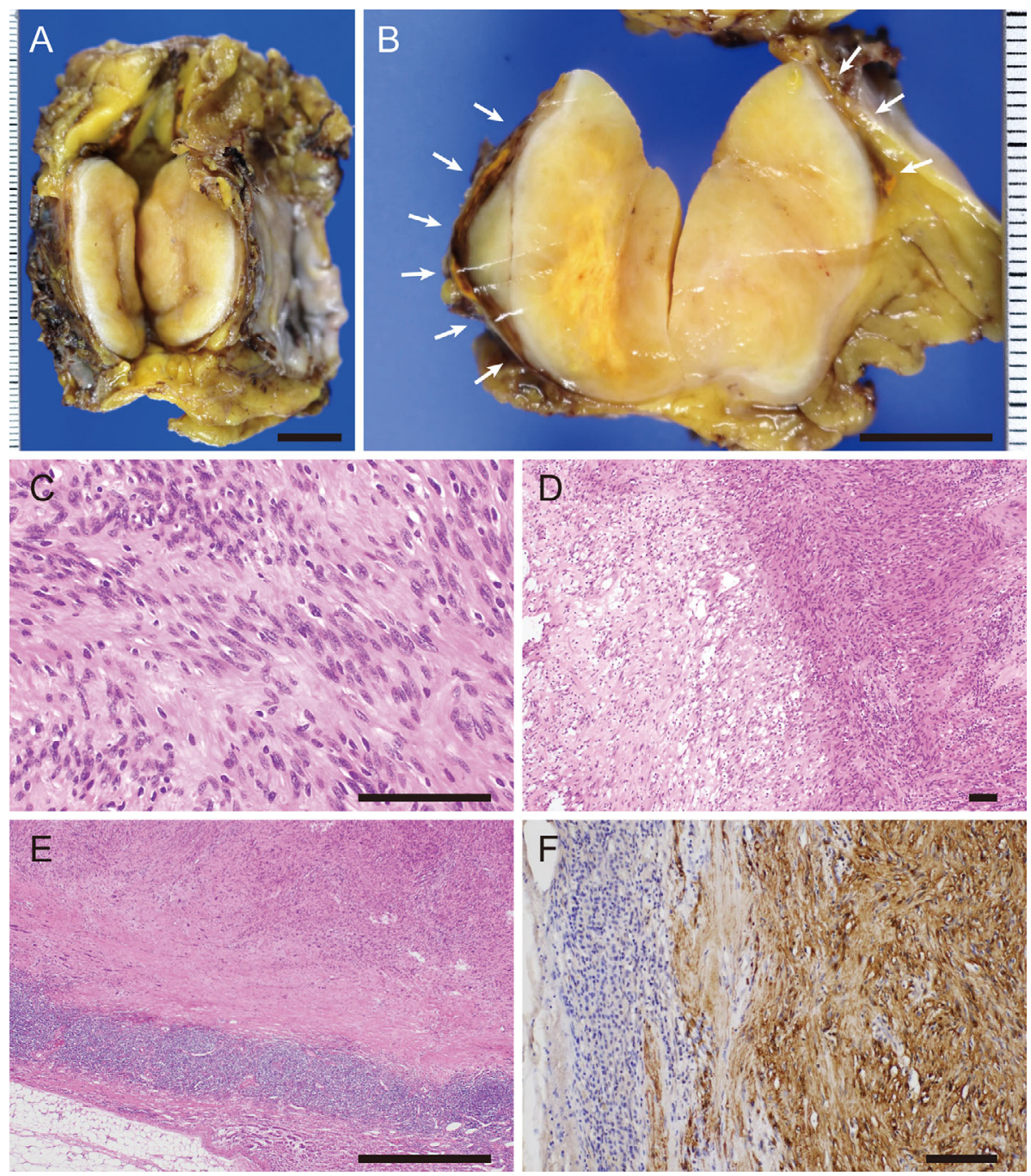

Figure 2. Macroscopic and microscopic findings of the adrenal schwannoma. Macroscopic findings of the adrenal schwannoma show a well-demarcated solid tumor with yellow-to-white cut surfaces (A). As indicated by the arrows, the tumor is located inside the adrenal gland (B). Hematoxylin and Eosin staining sections demonstrate nuclear palisading of plump spindle cells $(\mathrm{C})$, cellular and hypocellular lesions (D), and demarcation with peripheral fibrosis and lymphocytic infiltrates (E). Immunohistochemically, the tumor cells (right side) are diffusely positive for S100 protein (F). Scale bars in $A$ and $B$ represent $1 \mathrm{~cm}$. Scale bars in $C$, D, and $F$ represent $100 \mu \mathrm{m}$. The scale bar in $E$ represents 1 mm.

\section{Discussion}

We encountered an elderly man with schwannoma of the right adrenal gland. Schwannomas rarely arise from the adrenal glands, and only 80 cases of adrenal schwannomas have been reported thus far (1). Adrenal schwannomas comprise $0.7 \%$ of adrenal tumors, $1-3 \%$ of total schwannomas, and $0.4 \%$ of retroperitoneal tumors (1). Mohiuddin et al. reviewed 33 cases of adrenal schwannomas and reported a median patient age of 49 (range, 14-89) years old, a median tumor size of 5.5 (range, 0.6-14.5) cm, and a slight predominance in women (female-to-male ratio $=1.2: 1$ ) $(2)$. Another recent study including 31 cases of adrenal schwannoma reported a median patient age of 47 (range, 26-71) years old, with a median tumor size of 4.5 (range, 1.0-12.1) $\mathrm{cm}$ and a predominance in women (female-to-male ratio $=$ 1.4:1) (1). There have been no reported cases of adrenal schwannomas with malignant transformation (1). We believe that the case presented here involves the oldest patient with adrenal schwannoma, a condition with a good prognosis (1). In addition, the tumor was retrospectively evaluated using CT images obtained over the previous 10 years. The followup period was longer than that in a previous case report on adrenal schwannoma, which at the time had longest reported follow-up period (115 months) (1).

The clinical differential diagnosis of adrenal schwannoma includes solid adrenal gland tumors, such as ACA, ACC, metastatic tumor, adrenal myelolipoma, and ganglioneuroma. Pheochromocytoma is also included in the differential diagnosis of adrenal schwannoma, as pheochromocytoma can be asymptomatic (3), and adrenal schwannoma with catechola- 
mine hypersecretion has been reported (4). The tumor in our case could not be radiologically diagnosed as a schwannoma, possibly because of its rarity. A literature review revealed no preoperatively diagnosed cases of adrenal schwannoma. Most adrenal schwannomas are misdiagnosed as adrenal cortical adenomas. In our case, CT images of the tumor showed slow growth and good demarcation as well as a heterogeneous appearance, which is consistent with findings in schwannomas (5).

The MRI findings in our case were also compatible with those of retroperitoneal schwannomas, which typically show hypointensity on T1-weighted images and hyperintensity on T2-weighted images (6). Cystic degeneration can occur in schwannomas; however, the finding is not specific to adrenal schwannomas (7). Up to $70 \%$ of ACAs are lipid-rich and show low CT attenuation values of $\leq 10 \mathrm{HU}$. Thus, nonenhanced CT images in our case are not likely to have indicated the presence of ACA; however, the possibility of ACA could not be wholly denied, as $30 \%$ of ACAs are lipid-poor tumors that may show CT attenuation values of $>10 \mathrm{HU}$ (8). The MRI findings in the present patient resembled those of ACAs, as ACAs generally appear hypo- or iso-intense compared to the liver on T1-weighted images and hyper- or isointense compared to the liver on T2-weighted images (8). Although the use of CSMRI, which is used for the detection of lipid components with a decrease in signal intensity between in-phase and opposed-phase MRI scans, might have increased the sensitivity for hyperattenuating ACA (89\% and 75\% sensitivity for ACAs with 10-30 and 20-30 HU, respectively) (9), the tumor in our case did not show a significantly decreased signal intensity on CSMRI. The SUVmax on FDG-PET of the tumor was similar to that of nonfunctioning ACA (mean SUVmax: 3.2; range, 1.5-4.9) (10) and schwannoma (mean SUVmax: 3.7; range, 1.517.3) (11). Thus, ACA could not be ruled out in our case. ACA and some metastatic tumors, such as hepatocellular carcinomas and renal cell carcinomas, generally have microscopic lipid components, and CT and CSMRI findings of these metastatic cancers mimic ACA (12). Thus, a malignant tumor history and pathological diagnosis are necessary for the exclusion of these metastatic tumors.

Typical cases of ACCs result in an accurate radiological diagnosis, as ACCs are usually large tumors (average size, $9.8 \mathrm{~cm}$ ) with irregular tumor borders that may be necrotic, hemorrhagic, or calcified; however, ACCs can mimic the radiological findings of ACAs and adrenal myelolipomas (12). Torresan et al. reported that the SUVmax of FDG-PET could not differentiate non-functioning ACA from ACC (13).

A diagnosis of pheochromocytomas on imaging is frequently impossible because these lesions often undergo necrosis, fibrosis, and cystic and fatty degeneration. Pheochromocytomas typically do not show a decrease in signal intensity on CSMRI, but they can mimic ACAs, as pheochromocytomas may demonstrate fatty degeneration (12). The preoperative diagnosis of adrenal ganglioneuroma is extremely challenging, as it radiologically mimics pheochromocytoma,
ACA, and ACC $(14,15)$. Primary adrenal lymphoma can be confined to the adrenal gland and was considered a differential diagnosis in our case. Primary adrenal lymphomas have been reported to have a median Hounsfield density of 35 HU on CT, hypointensity on T1-weighted images, and hyperintensity on $\mathrm{T} 2$-weighted images, similar to our observed in case $(16,17)$. However, primary adrenal lymphoma was deemed unlikely, as the median largest dimension of primary adrenal lymphomas was $80 \mathrm{~mm}$ (range, 40-180 mm), the SUVmax of the tumor was high (median 17.24; range, 9.5-48), and extra-adrenal tumor localization was frequent (range, 68-77\%) (16,17). Adrenal myelolipoma was unlikely in our case because of the lack of macroscopic adipose tissue. Radiological distinction between adrenal schwannoma, ACA, ACC, pheochromocytoma, and ganglioneuroma appears to be impossible.

For incidentally discovered adrenal masses, surgical treatment of masses measuring $>4 \mathrm{~cm}$ is recommended $(18,19)$. In our case, surgical management of the tumor was considered because the tumor was expected to have grown to a size of $>4 \mathrm{~cm}$ and radiologically showed an indeterminate mass (20). Asymptomatic, non-functioning unilateral adrenal masses measuring $\leq 4 \mathrm{~cm}$ in diameter and with obviously benign imaging features do not require surgery (20). However, the imaging features of the tumor in our case were not obviously benign, as the CT attenuation value of 28.6 HU favored ACC rather than typical cases of ACA (13). Thus, adrenalectomy was considered a viable approach our case according to the 2016 guidelines for adrenal incidentalomas (20).

Histologically, the diagnosis of the tumor was not difficult because typical features of schwannomas were observed. Histological findings of adrenal schwannoma are similar to those of schwannomas arising from other sites (2). Among adrenal schwannomas, conventional schwannomas are the most common (1), and our case was also a case of conventional schwannoma. Benign neoplasms are also thought to have a low Ki-67 labeling index. The histological differential diagnoses of adrenal schwannomas include pheochromocytoma, ganglioneuroma, leiomyoma, solitary fibrous tumor, gastrointestinal stromal tumor, neurofibroma, and sustentaculoma $(2,21)$. Histological and immunohistochemical studies excluded these differential diagnoses.

\section{Conclusion}

We encountered a 91-year-old Japanese man with right adrenal schwannoma. To our knowledge, this report presents the oldest case of adrenal schwannoma to date. It also involves the longest follow-up of adrenal schwannoma thus far, with radiological images from the previous 10 years. No malignant changes were observed. A preoperative diagnosis failed to diagnose adrenal schwannoma. Although adrenal schwannomas are rare, they can be a differential diagnosis of ACA, ACC, pheochromocytoma, and ganglioneuroma. Despite the benign biological behavior and good prognosis, 
given the rarity of the tumor, follow-up studies on adrenal schwannomas are necessary.

The authors state that they have no Conflict of Interest (COI).

\section{Acknowledgement}

We thank Drs. Miho Tsutsui and Hinako Maruoka for their valuable comments and support. We thank Ms. Keiko Mizuno, Mr. Masahiko Ohara, Ms. Kaori Yasuoka, Ms. Yukari Wada, and Mr. Hiroyuki Tsutsui for preparing the histological and immunohistochemical specimens. We thank Editage (www.editage.jp) for the English language editing.

\section{Consent for publication}

Informed consent was obtained from the patient for publication of this case report and the use of accompanying images.

\section{References}

1. Zhou J, Zhang D, Li W, Zhou L, Xu H, Zheng S, et al. Primary adrenal schwannoma: a series of 31 cases emphasizing their clinicopathologic features and favorable prognosis. Endocrine 65: 662674, 2019.

2. Mohiuddin Y, Gilliland MG. Adrenal schwannoma: a rare type of adrenal incidentaloma. Arch Pathol Lab Med 137: 1009-1014, 2013.

3. Motta-Ramirez GA, Remer EM, Herts BR, Gill IS, Hamrahian $\mathrm{AH}$. Comparison of CT findings in symptomatic and incidentally discovered pheochromocytomas. AJR Am J Roentgenol 185: 684688, 2005.

4. Hou J, Zhang L, Guo Y, Chen H, Wang W. Primary adrenal schwannoma with catecholamine hypersecretion. Arch Med Sci 12: 681-683, 2016.

5. Kim SH, Choi BI, Han MC, Kim YI. Retroperitoneal neurilemoma: CT and MR findings. AJR Am J Roentgenol 159: 1023-1026, 1992.

6. Hayasaka K, Tanaka Y, Soeda S, Huppert P, Claussen CD. MR findings in primary retroperitoneal schwannoma. Acta Radiol 40: 78-82, 1999.

7. Dell'Aversano Orabona G, Ricci D, Emili I, Serpi F, Ferrara V, Vanzulli A. Adrenal schwannoma: a case report. BJR Case Rep 6: 20190044, 2020.

8. Terzolo M, Stigliano A, Chiodini I, Loli P, Furlani L, Arnaldi G, et al. AME position statement on adrenal incidentaloma. Eur J Endocrinol 164: 851-870, 2011.

9. Haider MA, Ghai S, Jhaveri K, Lockwood G. Chemical shift MR imaging of hyperattenuating ( $>10 \mathrm{HU})$ adrenal masses: does it still have a role? Radiology 231: 711-716, 2004.

10. Akkus G, Guney IB, Ok F, Evran M, Izol V, Erdogan S, et al. Diagnostic efficacy of 18F-FDG PET/CT in patients with adrenal incidentaloma. Endocr Connect 8: 838-845, 2019.

11. Miyake KK, Nakamoto $Y$, Kataoka TR, Ueshima C, Higashi T, Terashima T, et al. Clinical, morphologic, and pathologic features associated with increased FDG uptake in schwannoma. AJR American journal of roentgenology 207: 1288-1296, 2016.

12. Adam SZ, Nikolaidis P, Horowitz JM, Gabriel H, Hammond NA, Patel T, et al. Chemical shift MR imaging of the adrenal gland: principles, pitfalls, and applications. Radiographics 36: 414-432, 2016.

13. Torresan F, Crimi F, Ceccato F, Zavan F, Barbot M, Lacognata C, et al. Radiomics: a new tool to differentiate adrenocortical adenoma from carcinoma. BJS Open 5: 1-7, 2021.

14. Shawa H, Elsayes KM, Javadi S, Morani A, Williams MD, Lee $\mathrm{JE}$, et al. Adrenal ganglioneuroma: features and outcomes of 27 cases at a referral cancer centre. Clin Endocrinol (Oxf) 80: 342$347,2014$.

15. Mylonas KS, Schizas D, Economopoulos KP. Adrenal ganglioneuroma: what you need to know. World J Clin Cases 5: 373377, 2017.

16. Laurent C, Casasnovas O, Martin L, Chauchet A, Ghesquieres H, Aussedat $\mathrm{G}$, et al. Adrenal lymphoma: presentation, management and prognosis. QJM 110: 103-109, 2017.

17. Majidi F, Martino S, Kondakci M, Antke C, Haase M, Chortis V, et al. Clinical spectrum of primary adrenal lymphoma: results of a multicenter cohort study. Eur J Endocrinol 183: 453-462, 2020.

18. Young WF Jr. Clinical practice. The incidentally discovered adrenal mass. N Engl J Med 356: 601-610, 2007.

19. Zeiger MA, Thompson GB, Duh QY, Hamrahian AH, Angelos P, Elaraj D, et al. The American Association of Clinical Endocrinologists and American Association of Endocrine Surgeons medical guidelines for the management of adrenal incidentalomas. Endocr Pract 15 (Suppl 1): 1-20, 2009.

20. Fassnacht M, Arlt W, Bancos I, Dralle H, Newell-Price J, Sahdev A, et al. Management of adrenal incidentalomas: European Society of Endocrinology Clinical Practice Guideline in collaboration with the European Network for the Study of Adrenal Tumors. Eur J Endocrinol 175: G1-G34, 2016.

21. Lau SK, Romansky SG, Weiss LM. Sustentaculoma: report of a case of a distinctive neoplasm of the adrenal medulla. Am J Surg Pathol 30: 268-273, 2006.

The Internal Medicine is an Open Access journal distributed under the Creative Commons Attribution-NonCommercial-NoDerivatives 4.0 International License. To view the details of this license, please visit (https://creativecommons.org/licenses/ by-nc-nd/4.0/).
(C) The Japanese Society of Internal Medicine Intern Med Advance Publication 